\title{
Increased basic fibroblast growth factor release and proliferation in xenotransplanted squamous cell carcinoma after combined irradiation/anti-vascular endothelial growth factor treatment
}

\author{
KAI FRUTH ${ }^{1}$, STEFAN WEBER ${ }^{2}$, YUNUS OKCU ${ }^{1}$, RUEDIGER NOPPENS $^{4}$, \\ KLAUS U. KLEIN ${ }^{4}$, EVA JOEST ${ }^{3}$, JANA HEDRICH ${ }^{1}$, SEBASTIAN THILEMANN ${ }^{1}$, BENJAMIN POGORZELSKI ${ }^{1}$, \\ DIMITRIOS KOUTSIMPELAS ${ }^{1}$, STEFAN FISCHER ${ }^{2}$, KERSTIN MUENNEMANN $^{2}$, ANNETTE AFFOLTER ${ }^{1}$, \\ ULF R. HEINRICH $^{1}$, CHRISTOPH BROCHHAUSEN ${ }^{5}$, IRENE SCHMIDTMANN ${ }^{6}$, WOLF J. MANN ${ }^{1}$, \\ HEINZ SCHMIDBERGER ${ }^{3}$, LAURA M. SCHREIBER ${ }^{2}$ and JUERGEN BRIEGER ${ }^{1}$ \\ Departments of ${ }^{1}$ Otorhinolaryngology, Head and Neck Surgery, ${ }^{2}$ Radiology, ${ }^{3}$ Radiation Oncology and Radiation Therapy, \\ and ${ }^{4}$ Anaesthesiology, ${ }^{5}$ Institute of Pathology and ${ }^{6}$ Institute of Medical Biostatistics, Epidemiology and Informatics \\ (IMBEI), University Medical Center of the Johannes Gutenberg University Mainz, Mainz, Germany
}

Received December 14, 2011; Accepted January 9, 2012

DOI: $10.3892 / o r .2012 .1654$

\begin{abstract}
Novel strategies of cancer therapy combine irradiation and anti-angiogenic active compounds. However, little is known concerning the undesired cellular and molecular effects caused by this novel treatment concept. We used a mouse squamous cell carcinoma (SCC) xenotransplantation model to evaluate the potential undesired effects which compromise the success of this therapeutic combination. SCCs were subcutanously implanted in nude mice. Animals were treated with a fractionated irradiation scheme ( $5 \times 4 \mathrm{~Gy})$ alone or in combination with daily injections of anti-vascular endothelial growth factor (VEGF) antibodies. Controls remained untreated. Before and after treatment, resonance imaging (MRI), ultrasound and near-infrared spectrometry were used to evaluate tumor vessel integrity. Finally, tumors were explanted and VEGF, basic fibroblast growth factor (bFGF), vessel density, proliferation and apoptotic activity were analyzed by immunohistochemistry. Irradiation caused VEGF release and we found evidence for VEGF-mediated vessel protection. In the tumors derived from the combined treatment, blood volume was decreased, and apoptotic indices were increased. Remarkably, bFGF levels and proliferative indices were also increased. Combined irradiation/anti-VEGF treatment resulted in the desired VEGF depletion and increased tumor cell apoptosis. Nonetheless,
\end{abstract}

Correspondence to: Dr Juergen Brieger, Department of Otorhinolaryngology, Head and Neck Surgery, Laboratory of Molecular Tumorbiology, University Medical Center of the Johannes Gutenberg University Mainz, Langenbeckstr 1, D-55101 Mainz, Germany E-mail: juergen.brieger@unimedizin-mainz.de

Key words: squamous cell carcinoma, resistance, vascular endothelial growth factor, basic fibroblast growth factor, irradiation
bFGF and proliferation also increased, possibly suggesting a compensatory response. The application of additional targeted drugs may help develop more effective SCC treatments.

\section{Introduction}

The number of patients newly diagnosed with squamous cell carcinoma (SCC) is still increasing and the 5-year survival rate has remained largely unchanged $(1,2)$. Radiotherapy is an integral part of different SCC treatment protocols. However, frequently tumor response is insufficient. A multitude of underlying mechanisms are under discussion like decreased apoptosis induction, increased DNA repair capacity or the release of cytoprotective factors. To overcome resistance, novel treatment concepts targeting these mechanisms are urgently needed.

Tumor vascularization is closely related to therapy outcome and prognosis. Therefore, antiangiogenic treatment schedules alone or in combination with irradiation are under evaluation. The idea behind this concept is to normalize the dysfunctional tumor vasculature and/or to inhibit vessel regrowth or repair of the irradiation damaged tumor vasculature (3). However, several lines of evidence suggest that irradiation might induce additional undesired effects like the release of angiogenic growth factors, potentially impairing the expected irradiation effect (4). We have previosuly examined the induction of vascular endothelial growth factor (VEGF) and its receptors by irradiation and have reported that tumor-derived VEGF has cytoprotective effects on both tumor cells and on the endothelium (5-7).

Further challenges might arise from combined irradiation and antivascular treatment concepts. In glioblastoma, the induction of compensatory mechanisms in response to VEGF inhibition with emergence of upregulation of multiple proangiogenic and proinvasive factors during antivascular therapy has been reported (8). Accordingly, compensatory 
upregulation of the basic fibroblast growth factor (bFGF) after VEGF-depletion has been observed (9). However, the combined effects of both, antivascular treatment and irradiation, on tumor perfusion and vessel network integrity have not yet been addressed.

We analyzed xenotransplanted tumors by semi-quantitative immunohistochemistry and noninvasive monitoring techniques, such as magnetic resonance imaging (MRI), ultrasound and near infrared spectrometry $(\mathrm{O} 2 \mathrm{C})$ in order to identify molecular and functional changes generated after irradiation treatment alone or in combination with VEGF antibodies. Our data suggest that irradiation induces VEGF resulting in vessel protection. Combination of irradiation with anti-VEGF treatment seems to constrain VEGF action. However, the tumors release bFGF potentially compromising the success of the combination therapy.

\section{Materials and methods}

Cell culture. The HNCCUM-02T squamous cell carcinoma cell line was used (10). The cell line was established from a tumor resected from the base of the tongue and maintained in DMEM/ Ham's F12 (PAA, Pasching, Austria), supplemented with 5\% FCS (Greiner Frickenhausen, Germany) and antibiotics [penicillin $100 \mathrm{U} / \mathrm{ml}$ and streptomycin $100 \mu \mathrm{g} / \mathrm{ml},(\mathrm{PAA})]$ at $37^{\circ} \mathrm{C}$ in $5 \% \mathrm{CO}_{2}$.

Mouse experiments. To establish tumors, 6-week old nude mice (CD1-Nu/Nu, Charles River, Sulzfeld, Germany) were inoculated subcutaneously in both hind limbs with $1 \times 10^{7}$ cells each. The procedure has been detailed before (11). In total 18 animals were randomized, and 6 animals were assigned to each of the three groups: untreated controls, irradiation (IR)-treated and the IR-anti-VEGF-treated. One animal of the control group died before treatment started, therefore, this group comprised 5 animals. Tumor size and mouse body weight were measured twice weekly. Tumor volumes were calculated as $\mathrm{V}=\pi / 6 \mathrm{xab}^{2}$, where ' $\mathrm{a}$ ' stands for the longer and ' $b$ ' for the shorter diameter. After the tumors had reached an average diameter of 8-10 $\mathrm{mm}$ experiments were initiated. Tumors were analyzed by MRI, O2C and ultrasound imaging. Twenty-four hours after the initial imaging analysis radiation was delivered in five consecutive daily fractions using an X-ray unit for orthovoltage therapy (RT100; CHF Mueller, Hamburg, Germany). Homogenous dosages of 4 Gy daily were delivered with two opposing lateral fields. Forty-eight hours after the last irradiation treatment tumors were imaged again, mice were sacrificed, and tumors were resected and fixed in buffered formalin (4\%). Before irradiation and imaging, the mice were anesthetized by subcutaneous injection of $61.5 \mathrm{mg} / \mathrm{kg}$ ketamine (Ketamin-Ratiopharm, Ratiopharm, Ulm, Germany) and $2.3 \mathrm{mg} / \mathrm{kg}$ xylazine (Rompun $2 \%$, Bayer, Leverkusen, Germany). VEGF antibodies (RnD Systems, Wiesbaden, Germany) were applied i.p. (10 $\mu \mathrm{g} / \mathrm{animal}) 1 \mathrm{~h}$ prior to each irradiation. The treatment schedule is shown in Fig. 1A.

Mice were housed at the Research Animal Resource Center of the University Medical Center of the Johannes Gutenberg University Mainz. This facility is maintained in accordance with the regulations and standards of German law. Approval was obtained by the animal care committee.
Magnetic resonance imaging (MRI). All MRI examinations were performed on a whole body 1.5 T scanner (Siemens Magnetom Vision, Siemens Medical Solutions, Erlangen, Germany) equipped with a prototype gradient coil (gradient strength = $50 \mathrm{mT} / \mathrm{m}$, rise time $=300 \mu \mathrm{sec}$ ). For signal reception, an in-house developed 4-channel coil-array for simultaneous measurement of four mice was used. Radio frequency (RF) excitation was performed using the body coil of the MRI scanner. Tumor perfusion was determined using dynamic contrast enhanced (DCE) imaging and subsequent pharmacologic modeling using the Brix model (12). Therefore, $0.5 \mathrm{mmol} / \mathrm{kg}$ of body weight of Gd-DTPA (Magnevist,Bayer-Schering Pharma AG, Germany) were injected into the jugular vein. T1-weighted DCE imaging was performed using a fast spoiled gradient-echo pulse sequence with saturation recovery magnetization preparation (SR-TurboFLASH) with the following sequence parameters: time-to-saturation $(\mathrm{TI})=320 \mathrm{msec}, \mathrm{TR}=2.4 \mathrm{msec}, \mathrm{TE}=1.2 \mathrm{msec}$, flip angle, $18^{\circ} \mathrm{C}$. Each of the two acquired slices was positioned over one tumor, respectively. The spatial resolution was $1.2 \times 1.2 \times 4.0 \mathrm{~mm}^{3}$, the time resolution was $\sim 1 \mathrm{sec}$. One hundred and twenty images were acquired per slice. Data processing was performed with an in-house developed software in MATLAB (version 7.3.0; MathWorks, Inc., Natick, MA, USA). The data parameters of the model were set to values described in Pohlmann et al (13). Data analysis was performed on a pixel-by-pixel basis.

The mean perfusion value of the whole tumor was used by averaging all pixels in the tumor. Necrotic areas were excluded from analysis. The parameters A, which corresponds to the regional distribution of volume available for the contrast agent, and $k 21$, which describes the rate constant of contrast agent extravasation from intravascular to extravascular space, were determined and used for comparison with the other modalities.

Combined photo-spectrometry and laser Doppler flowmetry $(O 2 C)$. The underlying principle of regional capillary venous hemoglobin oxygen saturation $\left(\mathrm{srvO}_{2}\right)$ and regional capillary venous blood velocity (rvVelo) measurement by combined photo-spectrometry and laser-doppler flowmetry at the capillary venous level has been described in detail (14). We used the O2C-device (oxygen-to-see, LEA Medizintechnik GmbH, Giessen, Germany). In the present study, one single flat probe (type LF-2; probe head: width $12 \mathrm{~mm}$, height $5.5 \mathrm{~mm}$, length $44.5 \mathrm{~mm}$; probe length $300 \mathrm{~cm}$ ) was applied on the tissue measuring a banana-shaped volume in both 2 and $8 \mathrm{~mm}$ depth.

Ultrasound. We used a contrast enhanced ultrasound imaging technique. A Siemens Sonoline Elegra (Erlangen, Germany) ultrasound scanner was used, with a fixed linear array 7.5L40 transducer. Ultrasound device settings were as follows: Echo Contrast Imaging, mechanical index (MI) 0.1, transmit power $1 \%$ and gain $60 \mathrm{~dB}$. Image acquisition was started simultaneously with a contrast agent (SonoVue, Bracco, Milan, Italy) bolus injection (200 $\mu \mathrm{l})$. Images were saved as XLOOP files (Schuster \& Hewesta, XLOOP version 3.0, Bisamberg, Austria).

The parameters, time to peak (TTP) and maximum of intensity (max) were calculated to characterize tumor blood circulation and perfusion in a representative region of interest (ROI).

Tissue microarray and quantitative immunohistochemistry. From the resected tumor material, tissue microarrays (TMA) 
Table I. Primary and secondary antibodies with their dilutions used for immunohistochemistry.

\begin{tabular}{|c|c|c|c|c|}
\hline Primary antibody & Type & Dilution & Secondary antibody & Dilution \\
\hline $\begin{array}{l}\text { VEGF; Santa Cruz } \\
\text { Biotechnology, Inc. }\end{array}$ & Rabbit polyclonal IgG2 (clone A-20) & $1: 100$ & $\begin{array}{l}\text { Goat anti-rabbit-biotinylated; } \\
\text { Dako }\end{array}$ & $1: 250$ \\
\hline $\begin{array}{l}\text { bFGF; Santa Cruz } \\
\text { Biotechnology, Inc. }\end{array}$ & Rabbit polyclonal IgG (clone 147) & $1: 750$ & LSAB kit; Dako & - \\
\hline CD31; Abcam & $\begin{array}{l}\text { Rat monoclonal IgG2a } \\
\text { (clone rm0032-1d12) }\end{array}$ & $1: 50$ & $\begin{array}{l}\text { Rabbit anti-rat-biotinylated; } \\
\text { Dako }\end{array}$ & $1: 500$ \\
\hline Ki67; Dako & Mouse monoclonal IgG1 (clone MIB-1) & $1: 20$ & EnVision kit; Dako & - \\
\hline $\begin{array}{l}\text { Caspase 3; Cell } \\
\text { Signaling Technology }\end{array}$ & Rabbit polyclonal IgG & $1: 500$ & $\begin{array}{l}\text { Goat anti-rabbit-biotinylated; } \\
\text { Dako }\end{array}$ & $1: 250$ \\
\hline
\end{tabular}
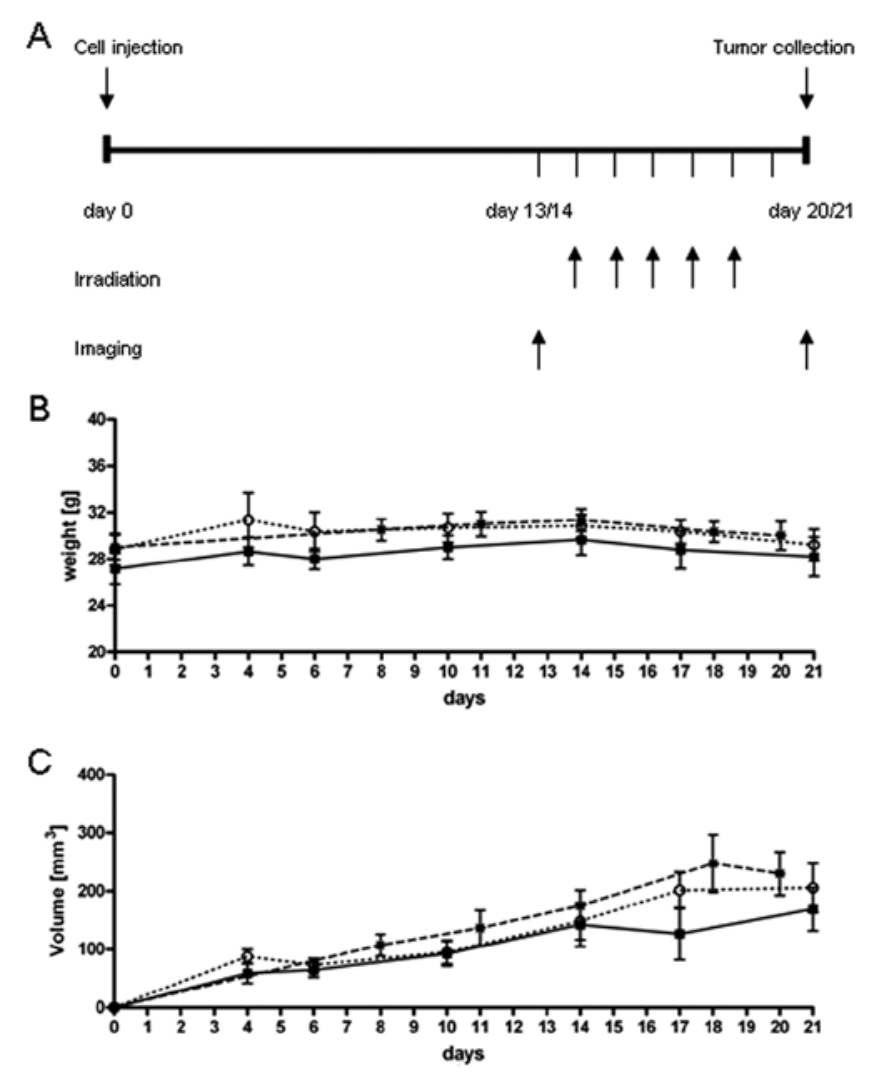

Figure 1. (A) Experimental schedule. (B) Mice weights. Shown are mean values $( \pm$ SEM). Animal weights were unchanged during therapy indicating no toxic effects. (C) Tumor sizes. Shown are mean values $( \pm$ SEM) of in minimum six tumors measured. No significant differences between the treatment groups were observed. a, control; O, IR; *, IR-anti-VEGF.

were created as detailed before (15). Immunohistochemical staining was performed by standard procedures. Sections incubated without the primary antibody served as negative and normal kidney tissue, angiofibroma or head and neck SCC as positive controls (data not shown) (for antibodies and dilutions please refer to Table I). Quantification of VEGF and bFGF was then performed using a computer-assisted approach as described in detail previously (16). Staining intensities are given as AU (arbitrary units). Quantification of proliferative activity (percent positive cells) and microvessel density (number of vessels, MVD) was performed as previously described $(17,18)$.

Statistics. Paired two-tailed t-tests were used for the comparison of the differences before and after treatment of the same tumors analyzed by the three imaging methods. Samples analyzed by quantitative immunohistochemistry were calculated by twotailed t-tests (unpaired). Differences with a P-value $\leq 0.05$ were considered statistically significant.

\section{Results}

Tumor growth. Animals were weighed twice weekly. Animal weights remained unchanged, indicating no serious nonspecific toxicological effects of the treatment (Fig. 1B). Additionally, tumor sizes were measured to calculate tumor volumes (Fig. 1C). No significant differences comparing the treatment groups were observed.

Imaging. To examine the hemodynamic functional changes of the tumor vasculature by a non-invasive method and to compare the available methods, we used MRI, O2C and ultrasound to measure several parameters before and after treatment. We analyzed 'A' and 'k21' by MRI (Fig. 2A and B). ' $A$ ' roughly represents a given blood volume of an analyzed tumor and indirectly represents its vessel density. In the irradiation group we found a slight increase of ' $A$ ' after irradiation treatment comparable to the control group. This is somewhat surprising as one would expect vessel damage after irradiation and thereby a decrease of 'A'. In the IR-anti-VEGF group we found a significant decrease of ' $A$ '. Both results indicate that irradiation results in lowered vessel density in the absence of VEGF and might suggest that in the IR group (without antiVEGF-antibodies) vessels are not damaged and eliminated due to a potentially irradiation-stimulated VEGF release and its protective activity.

' $\mathrm{k} 21$ ' provides a measure for the leakiness and thereby functionality of vessels. In the IR group we observed significantly increased ' $\mathrm{k} 21$ ' rates after treatment in comparison to the controls, indicating increased vessel permeability. This might be indicative of vessel damage due to irradiation. However, given that in the IR-anti-VEGF group decreased vessel permeability 
A

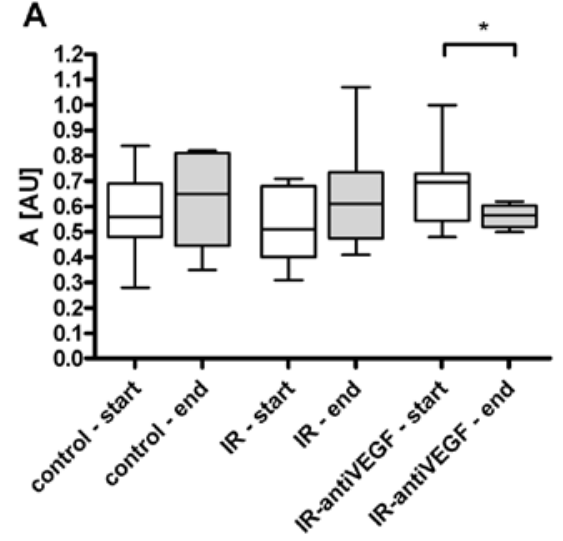

C

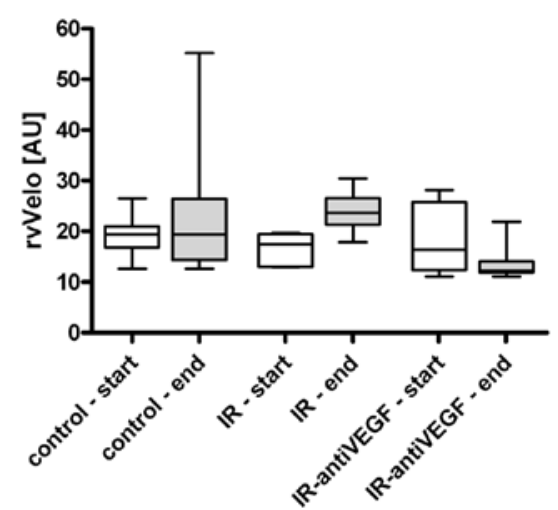

E

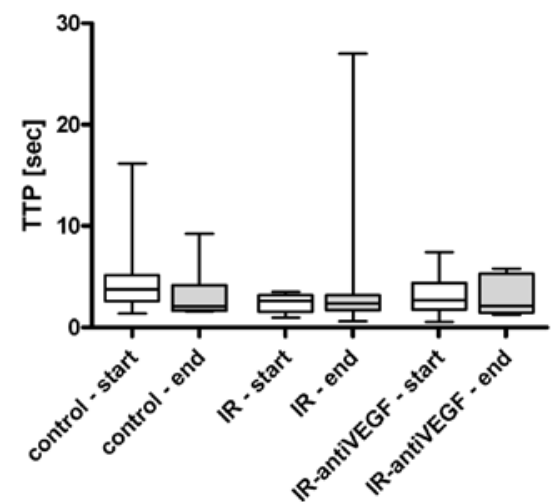

B

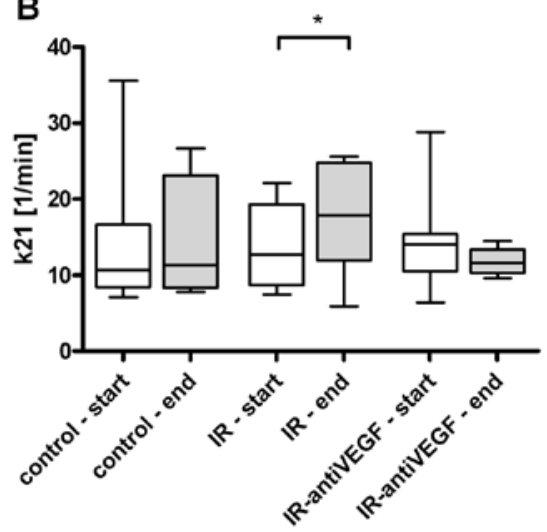

D

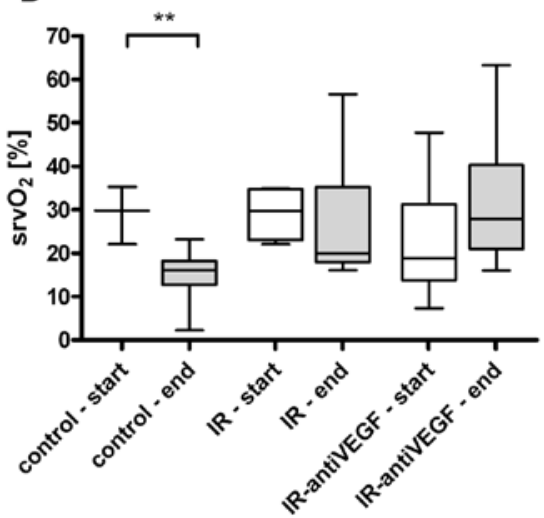

$\mathbf{F}$

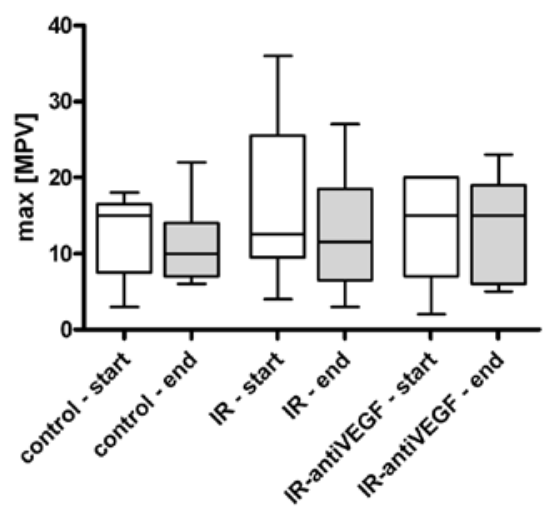

Figure 2. Hemodynamic parameters acquired by MRI (A and B), O2C (C and D), and ultrasound (E and F). Shown are mean values $( \pm$ SEM) of a minimum of three measured tumors. Differences were calculated by two tailed paired t-tests; ${ }^{*} \mathrm{P} \leq 0.05$ and ${ }^{* *} \mathrm{P} \leq 0.01$. (A) Blood volume was significantly decreased in the IR-anti-VEGF group. (B) In the same group decreased vessel permeability was observed after treatment, suggesting that after irradiation released VEGF mediates increased vessel permeability. (C) Accordingly, we observed increased venous blood flow velocity after irradiation and a non-significant decreased flow after IR-anti-VEGF treatment. (D) A tendency towards increased blood oxygenation after IR-anti-VEGF treatment has been observed, a notable observation especially in light of the significantly lowered levels in the controls and the IR group. (E and F) No changes could be identified by ultrasound imaging. (A) A, blood volume (AU, arbitrary units). (B) k21, exchange rate (1/min). (C) rvVelo, regional capillary venous velocity (AU, arbitrary units). (D) srvO ${ }_{2}$, regional capillary venous hemoglobin oxygen saturation (\%). (E) TTP, time to peak (sec). (F) max, maximal intensity (MPV, mean pixel value).

was observed after treatment, it may be that tumor-released VEGF (rather than irradiation damage) is the cause of increased vessel permeability in the IR group. This interpretation is supported by our data obtained by $\mathrm{O} 2 \mathrm{C}$ imaging. We observed increased venous blood flow velocity after irradiation and a non-significant decreased flow after IR-anti-VEGF treatment (Fig. 2C) likely due to an IR-induced secretion of VEGF and indicating an impact of VEGF on vasculature integrity. The data of the $2 \mathrm{~mm}$ measurement are shown. Those of the $8 \mathrm{~mm}$ measurement were comparable (data not shown).

The decrease of vascular permeability measured by MRI after the combined IR-anti-VEGF treatment (Fig. 2B) may be indicative for a resizing of the vasculature to a more functional type (vessel normalization). This assumption is supported by the oxygenation data obtained by near infrared imaging (Fig. 2D) suggesting a tendency towards increased blood 
A
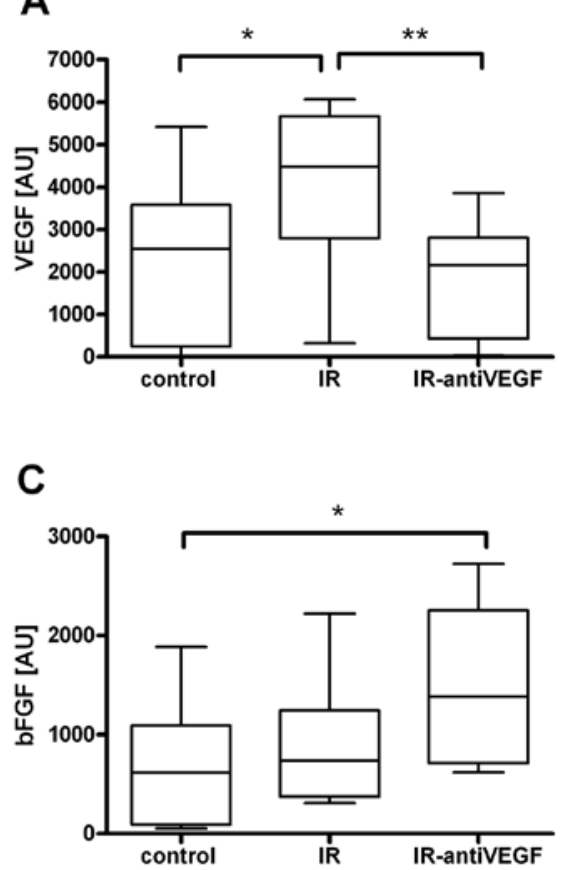

B

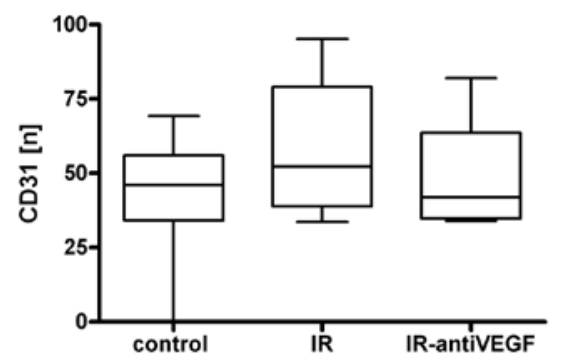

D

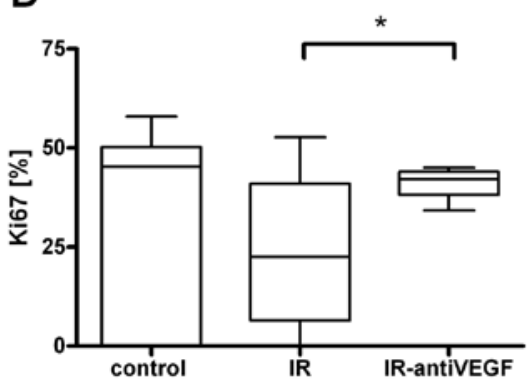

E

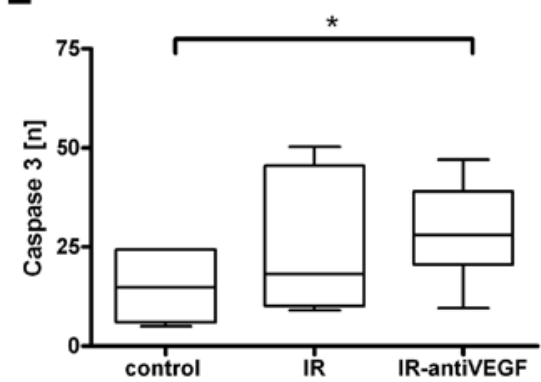

Figure 3. Immunohistochemical quantification of (A) VEGF, (B) CD31, (C) bFGF, (D) Ki67, (E) caspase 3. Shown are mean values ( \pm SEM) of a minimum of six tumors analyzed (x400). Increased levels of VEGF in the IR group were observed. (A) Anti-VEGF treatment resulted in the normalization of VEGF to pretreatment levels. (B) No changes of the vessel densities were observed. IR/anti-VEGF treatment resulted in strongly (C) increased bFGF-levels, (D) increased proliferative activity and (E) apoptosis. Differences were calculated by two tailed t-tests; ${ }^{*} \mathrm{P} \leq 0.05$ and ${ }^{* *} \mathrm{P} \leq 0.01$. VEGF, vascular endothelial growth factor (AU, arbitrary units); CD31, vessel marker (n=vessel count); bFGF, basic fibroblast growth factor (AU, arbitrary units); Ki67, marker of proliferating cells (positive nuclei); caspase 3, marker of apoptotic cells (positive cells).

oxygenation after IR-anti-VEGF treatment, a notable observation especially in light of the significantly lowered levels in the controls and the IR group. By ultrasound imaging we were not able to identify any significant changes after treatment (Fig. 2E and F). The assumption of IR-mediated VEGF release and associated VEGF-mediated cellular protection is supported by our immunohistochemical data.

Immunohistochemistry. To examine the cellular and molecular changes of the xenotransplanted tumors in response to combined IR-anti-VEGF treatment or IR alone, we evaluated the expression of several potentially implicated proteins (Figs. 3 and 4).

In the irradiation group we observed a significant increase of released VEGF, and decreased levels in the IR-anti-VEGF group compared to the irradiated group suggesting the release of VEGF after irradiation and a VEGF neutralizing activity of the anti-VEGF treatment (Fig. 3A). This observation is in accordance with our MRI and $\mathrm{O} 2 \mathrm{C}$ results where we found evidence for a cytoprotective activity potentially mediated by IR-stimulated released VEGF. According to the increased VEGF levels after irradiation, no decrease of vessel density was observed as one would have expected after irradiation. Remarkably, in the IR-anti-VEGF group the vessel count was not significantly decreased (Fig. 3B). This might be due to the observed increased bFGF levels observed in this group (Fig. 3C). High bFGF levels were accompanied by heightened proliferative activity in the IR-anti-VEGF group compared to the IR group. (Fig. 3D). Additionally, in this cohort the highest apoptotic indices were observed (Fig. 3E), indicating that antiVEGF treatment in combination with IR is efficient in respect to induction of apoptosis but not in controlling proliferation.

\section{Discussion}

To enhance the efficacy of conventional anticancer therapies and to overcome tumor resistance, the combination of irradiation with biologicals is currently under evaluation $(19,20)$. However, compensatory mechanisms after irradiation and the 


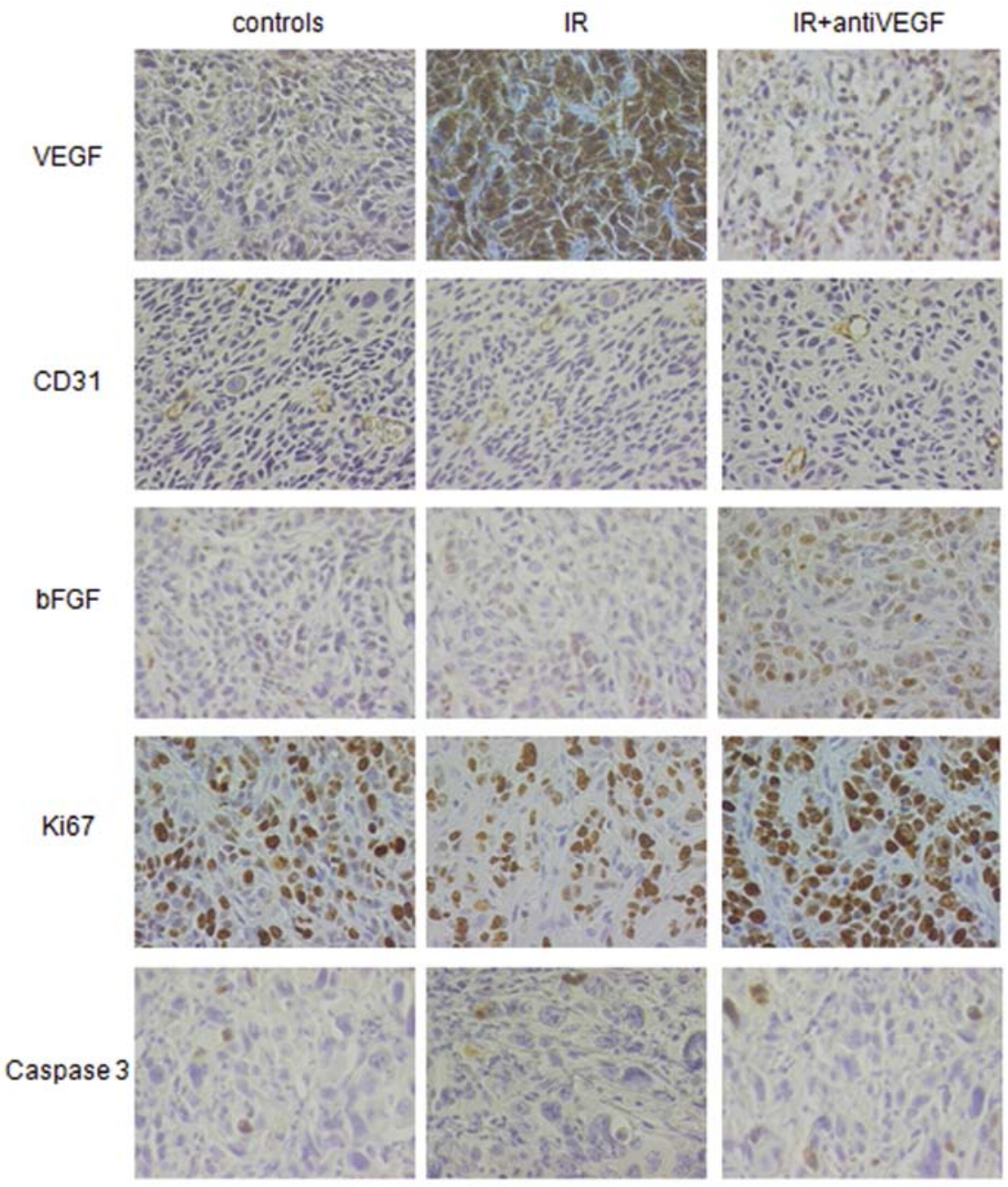

Figure 4. Representative immunohistochemical stainings of VEGF, CD31, bFGF, Ki67 and caspase 3.

potential therapeutic success-limiting tumor responses to the combined treatment remain largely unknown. Therefore, we utilized a mouse SCC-xenotransplant model to evaluate the underlying mechanisms in vivo.

Concerning the tumor volume we observed only slight differences between the treatment groups. This observation is in line with our expectations as significant tumor size changes were not anticipated shortly after treatment cessation (21). Mice weights were unaffected as well, indicating that the observed effects on the tumors were not due to nonspecific toxic effects of the treatment. However, we observed cellular changes with potential consequences on tumor architecture maintenance and therapeutic success occurring prior to macroscopic manifestation by functional imaging. These effects were confirmed by immunohistochemistry. After combined IR-antiVEGF treatment we found evidence for reduced expression of cellular VEGF followed by reduced tumor blood volume and vessel permeability, but increased tissue oxygenation. These data are in accordance with and support the concept of vessel normalization by antiangiogenic treatment introduced by Winkler et al (22). They suggest that antiangiogenic treatment results in decreased vessel counts but in an improvement of vessel function. This 'vessel normalization' might be beneficial in a combined treatment setting as increased tissue oxygenation and reduced interstitial fluid pressure due to reduced leakiness of the vessels (23) should increase efficiency of irradiation therapy or chemotherapeutic drug uptake.

Our group has previously examined the in vitro release of VEGF after irradiation. This release results in increased irradiation resistance of treated tumor cells $(7,24)$. We now found evidence that VEGF is similarly released in vivo from irradiated tumors, an observation of special interest in fractionated therapeutic settings. According to our data, the released VEGF obviously mediates physiological responses of the tumor, such as vessel density, apoptosis, and vessel functionality, features which are potentially relevant for treatment response. The IR-anti-VEGF treatment exerts effects on the tumor morphology which may increase the success of the therapy. However, we observed additional unexpected and undesired responses, such as increased proliferative activity and increased bFGF levels in the IR-anti-VEGF group. Increased proliferation after antiangiogenic therapy has been reported before (25). The authors speculated this increase to reflect an improved tumor microenvironment due to vessel normalization. We suppose that the bFGF upregulation is a compensatory reaction of the tumor due to VEGF depletion. The promoted proliferative activity in this group may in turn be related to the observed increased bFGF release rather than to a generally 'improved microenvironment'. 
The partly unexpected results demonstrate that the use of specific antiangiogenic active compounds might result in effects potentially compromising the initial positive treatment response. One might speculate that these effects could be overcome by a more sophisticated treatment regimen including additional anti-bFGF treatment. Another option might be the use of less specific but more global acting drugs like tyrosin kinase inhibitors thereby targeting multiple signaling pathways activated by irradiation and inhibiting a multitude of downstream effects.

In consequence there is a great need to establish reliable biomarkers to monitor individual clinical responses to novel combined treatment concepts. Techniques for close monitoring of tumor responses might be helpful for appropriate therapy and drug scheduling. Several imaging methods of angiogenic vasculature have been developed in the past (26). According to our experience functional MRI or O2C are easy, fast, and cost-effective techniques that have the potential to monitor tumor response. In our setting ultrasound imaging was not sensitive and reliable enough for the assessment of the functional changes after treatment.

The present analysis provides important data about the compensatory responses to a combined IR-anti-VEGF therapy of SCC. After IR-anti-VEGF treatment we observed the desired (increased programmed cell death) and undesired (increased bFGF-levels and proliferative activity) effects in our model, potentially accounting for unsatisfying results of novel treatment concepts. Our data suggest the need for the inhibition of cytoprotective factors and of factors released in a compensatory loop to overcome resistance mechanisms. Therefore, novel combinations of biologicals like anti-bFGF and anti-VEGF in concert with irradiation might be beneficial. Non-invasive imaging techniques should be considered to monitor cellular and treatment responses.

\section{Acknowledgements}

We are greatly indebted to Simone Mendler and Karin Bakes for their excellent technical assistance and Dr Herbert Schuster for helpful discussions and software support. This study was funded by a grant provided by the Foundation Tumour Research Head and Neck, Wiesbaden, Germany. The foundation is a non-profit organization. The funders played no role in the experimental design, execution, analysis or preparation of the manuscript.

\section{References}

1. Kim MM and Califano JA: Molecular pathology of head-andneck cancer. Int J Cancer 112: 545-553, 2004.

2. Jemal A, Siegel R, Ward E, et al: Cancer statistics. CA Cancer J Clin 58: 71-96, 2008.

3. Horsman MR, Siemann DW: Pathophysiologic effects of vasculartargeting agents and the implications for combination with conventional therapies. Cancer Res 66: 11520-11539, 2006.

4. Gorski DH, Beckett MA, Jaskowiak NT, et al: Blockage of the vascular endothelial growth factor stress response increases the antitumor effects of ionizing radiation. Cancer Res 59: 3374-3378, 1999.

5. Koutsimpelas D, Brieger J, Kim DW, Stenzel M, Hast J and Mann WJ: Proangiogenic effects of ionizing irradiation on squamous cell carcinoma of the hypopharynx. Auris Nasus Larynx 35: 369-375, 2008
6. Brieger J, Schroeder P, Gosepath J and Mann WJ: VEGF-subtype specific protection of SCC and HUVECs from radiation induced cell death. Int J Mol Med 15: 145-151, 2005.

7. Brieger J, Schroeder P, Gosepath J and Mann WJ: Vascular endothelial growth factor and basic fibroblast growth factor are released by squamous cell carcinoma cells after irradiation and increase resistance to subsequent irradiation. Int J Mol Med 16: 159-164, 2005

8. Lucio-Eterovic AK, Piao Y and de Groot JF: Mediators of glioblastoma resistance and invasion during antivascular endothelial growth factor therapy. Clin Cancer Res 15: 4589-4599, 2009.

9. Dempke WC and Heinemann V: Resistance to EGF-R (erbB-1) and VEGF-R modulating agents (Review). Eur J Cancer 45: 1117-1128, 2009.

10. Welkoborsky HJ, Jacob R, Riazimand SH, Bernauer HS and Mann WJ: Molecular biologic characteristics of seven new cell lines of squamous cell carcinomas of the head and neck and comparison to fresh tumor tissue. Oncology 65: 60-71, 2003.

11. Vlachtsis K, Brieger J, Kim DW, et al: Quantitative analysis of VEGF-isoforms in head and neck squamous cell carcinoma cell lines: relation to xenotransplantability and tumour progression in mice. Oncol Rep 9: 1133-1138, 2002.

12. Hoffmann U, Brix G, Knopp MV, Hess T and Lorenz WJ: Pharmacokinetic mapping of the breast: a new method for dynamic MR mammography. Magn Reson Med 33: 506-514, 1995.

13. Pohlmann A, Moller M, Decker H and Schreiber WG: MRI of tarantulas: morphological and perfusion imaging. Magn Reson Imaging 25: 129-135, 2007.

14. Klein KU, Schramm P, Glaser M, et al: Intraoperative monitoring of cerebral microcirculation and oxygenation - a feasibility study using a novel photo-spectrometric laser-Doppler flowmetry. J Neurosurg Anesthesiol 22: 38-45, 2010.

15. Affolter A, Fruth K, Brochhausen C, Schmidtmann I, Mann WJ and Brieger $\mathrm{J}$ : Activation of mitogen-activated protein kinase extracellular signal-related kinase in head and neck squamous cell carcinomas after irradiation as part of a rescue mechanism. Head Neck 33: 1448-1457, 2011.

16. Selivanova O, Heinrich UR, Brieger J, Feltens R and Mann W: Fast alterations of vascular endothelial growth factor (VEGF) expression and that of its receptors (Flt-1, Flk-1 and Neuropilin) in the cochlea of guinea pigs after moderate noise exposure. Eur Arch Otorhinolaryngol 264: 121-128, 2007.

17. Bedavanija A, Brieger J, Lehr HA, Maurer J and Mann WJ: Association of proliferative activity and size in acoustic neuroma: implications for timing of surgery. J Neurosurg 98: 807-811, 2003.

18. Brieger J, Bedavanija A, Lehr HA, Maurer J and Mann WJ: Expression of angiogenic growth factors in acoustic neurinoma. Acta Otolaryngol 123: 1040-1045, 2003

19. Bernier J, Bentzen SM and Vermorken JB: Molecular therapy in head and neck oncology. Nat Rev Clin Oncol 6: 266-277, 2009.

20. Riesterer O, Milas L and Ang KK: Combining molecular therapeutics with radiotherapy for head and neck cancer. J Surg Oncol 97: 708-711, 2008.

21. Hast J, Schiffer IB, Neugebauer B, et al: Angiogenesis and fibroblast proliferation precede formation of recurrent tumors after radiation therapy in nude mice. Anticancer Res 22: 677-688, 2002.

22. Winkler F, Kozin SV, Tong RT, et al: Kinetics of vascular normalization by VEGFR2 blockade governs brain tumor response to radiation: role of oxygenation, angiopoietin-1, and matrix metalloproteinases. Cancer Cell 6: 553-563, 2004.

23. Tong RT, Boucher Y, Kozin SV, Winkler F, Hicklin DJ and Jain RK: Vascular normalization by vascular endothelial growth factor receptor 2 blockade induces a pressure gradient across the vasculature and improves drug penetration in tumors. Cancer Res 64: 3731-3736, 2004.

24. Brieger J, Kattwinkel J, Berres M, Gosepath J and Mann WJ: Impact of vascular endothelial growth factor release on radiation resistance. Oncol Rep 18: 1597-1601, 2007.

25. Willett CG, Boucher Y, Duda DG, et al: Surrogate markers for antiangiogenic therapy and dose-limiting toxicities for bevacizumab with radiation and chemotherapy: continued experience of a phase I trial in rectal cancer patients. J Clin Oncol 23: 8136-8139, 2005.

26. Miller JC, Pien HH, Sahani D, Sorensen AG and Thrall JH: Imaging angiogenesis: applications and potential for drug development. J Natl Cancer Inst 97: 172-187, 2005. 